\title{
Reversion of antibiotic resistance in Mycobacterium tuberculosis by spiroisoxazoline SMARt-420
}

Nicolas Blondiaux ${ }^{*, 1}$, Martin Moune ${ }^{*, 1}$, Matthieu Desroses ${ }^{*, 2,3}$, Rosangela Frita ${ }^{* 1}$, Marion Flipo ${ }^{2}$, Vanessa Mathys $^{4}$, Karine Soetaert ${ }^{4}$, Mehdi Kiass ${ }^{4}$, Vincent Delorme ${ }^{1,5}$, Kamel Djaout ${ }^{1}$, Vincent Trebosc ${ }^{6,7}$, Christian Kemmer $^{6}$, René Wintjens ${ }^{8}$, Alexandre Wohlkönig ${ }^{9,10}$, Rudy Antoine ${ }^{1}$, Ludovic Huot ${ }^{1}$, David Hot ${ }^{1}$, Mireia Coscolla $^{11,7}$, Julia Feldmann ${ }^{11,7}$, Sebastien Gagneux ${ }^{11,7}$, Camille Locht ${ }^{1}$, Priscille Brodin ${ }^{1}$, Marc Gitzinger ${ }^{6}$, Benoit Déprez $z^{\#, 2}$, Nicolas Willand ${ }^{* \#, 2}$, Alain R. Baulard ${ }^{*, \#, 1}$

\section{Equivalent*}

\section{Corresponding\#}

1. Institut Pasteur de Lille, Univ. Lille, CNRS, Inserm, CHU Lille, U1019-UMR8204-CIIL-Centre d'Infection et d'Immunité de Lille, F-59000 Lille, France

2. Univ. Lille, Inserm, Institut Pasteur de Lille, U1177 - Drugs and Molecules for living Systems, F59000 Lille, France

3. Division of Translational Medicine and Chemical Biology, Science for Life Laboratory, Department of Medical Biochemistry and Biophysics, Karolinska Institutet, Stockholm, Sweden

4. National Reference Center for Tuberculosis and Mycobacteria, Bacterial Diseases Service, Operational Direction Communicable and Infectious Diseases, Scientific Institute of Public Health (WIV-ISP), Brussels, Belgium

5. Tuberculosis Research Laboratory, Institut Pasteur Korea, South Korea

6. Bioversys AG, Hochbergerstrasse 60C, 4057 Basel, Switzerland

7. University of Basel, Basel, Switzerland

8. Laboratoire des biopolymères et des nanomatériaux supramoléculaires, Université Libre de Bruxelles, Brussels, Belgium

9. VIB Center for Structural Biology, VIB, Pleinlaan 2, 1050 Brussels, Belgium

10. Structural Biology Brussels, Vrije Universiteit Brussel (VUB), Pleinlaan 2, 1050 Brussels, Belgium

11. Swiss Tropical and Public Health Institute, Basel, Switzerland 


\begin{abstract}
Antibiotic resistance is one of the biggest threats to human health globally. Alarmingly, multi- and extensively-drug resistant $M$. tuberculosis have now spread worldwide. Some key antituberculosis antibiotics are prodrugs, for which resistance mechanisms are mainly driven by mutations in the bacterial enzymatic pathway required for their bioactivation. We have developed drug-like molecules that activate a cryptic alternative bio-activation pathway of ethionamide in $M$. tuberculosis thereby circumventing the classic activation pathway where resistance mutations have now been observed. The first of its kind molecule, named SMARt-420 (ㅁmall Molecule $\underline{\text { Aborting }}$ Resistance), not only fully reverses ethionamideacquired resistance and clears ethionamide-resistant infection in mice, but also increases the basal sensitivity of bacteria to ethionamide.
\end{abstract}


Antibiotic resistance is a rapidly growing health concern and is observed for many anti-bacterial agents, both in hospital and in community settings $(1,2)$.

The development of drug resistance, especially rifampicin resistance (RR), multi-drug resistance (MDR) and extensive drug-resistance (XDR), is particularly worrisome for tuberculosis (TB) (3). Approximately 580,000 MDR/RR-TB cases have occurred in 2015, resulting in about 250,000 deaths. This situation seriously undermines efforts to control the global epidemic of TB and may soon counteract the slow but continuous annual decline of $\sim 1.5 \%$ observed during the last 14 years (4).

Discovering new anti-TB therapeutics is difficult (5), and few new drugs have emerged during the past 30 years. Moreover, as current TB treatment requires poly-therapeutic approaches, losing key antibiotics because of emergence of drug-resistance may impair the efficacy of the whole combination.

Drug resistance in bacteria can occur by mutations in the antibiotic's target, by the acquisition of enzymes that modify or degrade the drug, such as aminoglycoside-modifying enzymes or $\beta$-lactamases, by their active expulsion from the bacteria, or by alterations of the cell permeability (6). Sometimes, antibiotic resistance can be reversed, as for example by restoration of the anti-microbial activity of $\beta$-lactams by clavulanic acid that inhibits enzymes responsible for their degradation (7). Unfortunately, there are so far no other examples of this 40 year-old paradigm.

Some of the most effective anti-TB antibiotics require bio-activation by Mycobacterium tuberculosis enzymes to acquire their antibacterial effect. These pro-antibiotics not only include the 40 year-old compounds isoniazid (INH), pyrazinamide (PZA), p-aminosalicylic acid (PAS) and ethionamide (ETH), but also the recently approved drug delamanid (OPC-67683) and compounds under development, such as pretomanid (PA824) and TBA-354. However, bioactivation of pro-antibiotics is vulnerable to mutational inactivation or attenuation of the corresponding bio-activating enzymes, as observed for INH-, PZA-, and ETH-resistant clinical isolates with mutations in $\operatorname{katG}(8), \operatorname{pncA}(9)$, and ethA $(10,11)$, respectively. Similarly, experimentally generated and clinical resistance to delamanid and to pretomanid pointed to 
enzymes and co-enzymes involved in their bio-activation (12-14). Resistance to PAS also involves mutations in enzymes, such as dihydrofolate synthase, which is implicated in its bioactivation(15).

We have discovered a spiroisoxazoline family of $\underline{S}$ mall $\underline{\text { Molecules }}$ Aborting Resistance (SMARt) that induces expression an alternative bio-activation pathway of ETH, thereby reverting acquired resistance of M. tuberculosis to this antibiotic.

The bio-activation of ETH in M. tuberculosis is normally catalyzed by the Baeyer-Villiger monooxygenase EthA $(10,11,16)$. Transformation of ETH by EthA into highly reactive intermediates leads to the formation of a stable covalent adduct of ETH to nicotinamide adenine dinucleotide (NAD) $(10,17)$. This adduct binds to and inhibits the enoyl reductase InhA involved in mycolic acid biosynthesis, one of the essential components in the mycobacterial cell wall $(18,19)$. The production of EthA is regulated by the TetR-type transcriptional repressor EthR (20). Previously, we have shown that small molecule inhibitors of EthR stimulate the transcription of the ethA gene (21-24), which improves the bio-activation of ETH and consequently boosts its antibiotic activity, both in vitro and in vivo (25). These booster molecules, such as BDM41906, reduce, or reset the innate resistance of $M$. tuberculosis to ETH; however, as expected, they were unable to boost the bio-activation of ETH in strains harboring mutations in ethA (Table 1, panel B).

During optimization of first-generation EthR inhibitors, most derivatives revealed a good correlation between binding to EthR and ETH boosting activity against the bacteria (21-24). However, unexpectedly, the replacement of the oxadiazole-piperidine motif by a more constrained, structurally divergent, spiroisoxazoline scaffold completely abolished the ability of the compounds to bind to EthR in vitro, whereas they remained highly effective in boosting ETH activity against M. tuberculosis (Fig.1A).

As these compounds had no antibacterial activity in the absence of ETH but boosted ETH activity independently of EthR, we hypothesized that they may trigger an alternative bio-activation pathway for ETH. 
To identify this pathway, we studied the impact of SMARt-420, a representative member of the spiroisoxazoline family, on the transcriptome of $M$. bovis BCG, and compared it with the impact of the bona fide EthR-inhibitor BDM41906.

When the mycobacteria were treated with BDM41906, overexpression of both ethA and ethR was observed (Fig. 1B), in agreement with previous reports showing that EthR represses both ethA and its own expression (20). No other major modification of the transcriptome was observed, suggesting that the inhibitory activity of BDM41906 is restricted to EthR. In contrast to BDM41906, SMARt-420 only weakly induced the expression of ethA and ethR. However, SMARt-420 strongly activated the expression of the distantly located group of genes bcg_0107c-bcg_0108c, corresponding to rv0076c-rv0077c (Fig.1C) in $M$. tuberculosis. Based on protein homology, Bcg_0108c is predicted to be a member of the large family of oxidoreductases (http://enzyme.expasy.org/EC/1.-..-), which also includes EthA. Strikingly, in silico analyses revealed that $b c g \_0108 c$ is neighboring the tetR type transcriptional regulator gene bcg_0109 (http://pfam.xfam.org/family/PF00440), indicating analogies between the $r v 0076 c-r v 0078$ and the ethRethA loci (rv3854c-rv3855). The genetic organization of the two loci is also similar: $r v 0077 c$ and $r v 0078$, like ethA and ethR, are divergent open reading frames, both separated by small intergenic regions (76 bp and $62 \mathrm{bp}$ for ethR-ethA and rv0077c-rv0078, respectively) (Fig.1C). By analogy with the transcriptional organization of the ethA-ethR regulon (20), these observations indicate that Rv0078 might regulate the expression of $r v 0077 c$ by binding within the intergenic region. This hypothesis was confirmed by surface plasmon resonance experiments (SPR, Biacore ${ }^{\circledR}$ ) demonstrating the specific binding of Rv0078 to the intergenic region of rv0077c-rv0078 (Fig. 2A). In contrast, no binding of EthR to the intergenic region of rv0077c-rv0078 was observed, even at high concentration of protein, thus excluding EthR for controlling the expression of the rv0076-rv0078 locus (Fig. 2B). Conversely, no interaction was detected between Rv0078 and the ethA-ethR intergenic region, indicating the absence of crosstalk between the two regulons (Fig. 2B). We assigned the names EthR2 and EthA2 to Rv0078 and Rv0077c, respectively.

The direct binding of SMARt-420 to EthR2 was analyzed in vitro by thermal shift assay. Figure $2 \mathrm{C}$ illustrates the dose dependent thermal stabilization of EthR2 through binding to SMARt-420, whereas no 
interaction between BDM41906 and EthR2 was observed at equivalent concentrations, which is in agreement with the lack of effect of BDM41906 on the transcription of ethA2.

A detailed understanding of the interaction of SMARt-420 with EthR2 was obtained by the X-ray structure of the complex, which shows that EthR2 forms a homodimer in which one molecule of SMARt-420 is embedded in each monomer (Fig. 2D). The structure also confirmed EthR2 as a typical TetR-type regulator harboring two twistable helix-turn-helix $(\mathrm{HTH})$ motifs typically involved in the binding of the homodimer to its DNA target (26). In this family of repressors, binding of ligands to the distant specific pocket located in the upper part of the homodimer induces allosteric reorganization of the architecture of the HTH motifs, and thereby modifies the binding properties of the protein to its DNA target (23). In agreement with this paradigm, the EthR2-SMARt-420 co-crystal revealed that the distance separating the HTH motifs is far larger than the $\pm 34 \AA$ required for the binding of the regulator to DNA (27-29), thus providing the mechanism of action of SMARt-420 (Fig. 2D).

To quantify the inhibition of EthR2-binding to its DNA target by SMARt-420, we designed a synthetic mammalian gene circuit that senses the EthR2-DNA interaction in human cells and produces a quantitative reporter gene expression readout (SEAP, secreted alkaline phosphatase) (30) (Fig.3A). In contrast to its repressor role in mycobacteria, binding of EthR2 to the chimeric promoter used in this assay is expected to activate the expression of the SEAP reporter gene. In the absence of SMARt-420, we observed strong SEAP production, confirming the binding of EthR2 to its DNA promoter in this cellular context. Upon adding SMARt-420, a dose-dependent inhibition of SEAP production was observed, confirming that SMARt-420 impairs the DNA binding properties of EthR2. In contrast and as expected, no effect was observed when the cells were incubated with BDM41906, confirming the specificity of the SMARt-420-EthR2 interaction (see Fig.3A). In vitro, SPR experiments showed that SMARt-420 inhibits in a dose dependent manner the binding of EthR2 to its DNA target (Fig.3B) thus confirming that no other partner is required. 
The expression of ethA2 upon inhibition of EthR2 by SMARt-420 leads to efficient bio-activation of ETH in the bacteria. To evaluate the role of EthA2 in the SMARt-420-controled bio-activation of ETH, $M$. tuberculosis H37Rv was engineered to overexpress ethA2 using the multi-copy plasmid pMV261 (31). Under these conditions, the minimal inhibitory concentration (MIC) of ETH decreased from $2 \mu \mathrm{g} / \mathrm{ml}$ to 0.25 $\mu \mathrm{g} / \mathrm{ml}$, suggesting that EthA2 takes part in the bio-activation of ETH when overexpressed, thus reducing the innate resistance of the bacteria to ETH (Fig.3C).

SMARt-420 is the most active compound of a spiroisoxazoline series for which the binding to EthR2 (Thermal shift assay) and the inhibition of the DNA binding of EthR2 (SEAP assay) were shown to be correlated to the ETH boosting effect on sensitive and on ETH resistant $M$. tuberculosis. (Fig. S2).

As indicated by the transcriptomic analyses, the basal expression level of ethA2 in the absence of SMARt420 is low in M. tuberculosis. We measured the relative abundance of mRNA by high-throughput RNAsequencing in M. tuberculosis cells grown to mid-log phase. We found that ethA2 belongs to the $10 \%$ least-expressed genes in M. tuberculosis (rank 3609 out of the 3973 Tb genes), whereas ethA is among the $10 \%$ genes with the highest levels of expression. When compared to each other, ethA2-mRNAs were about 60 times less abundant than ethA-mRNAs in M. tuberculosis grown under standard conditions. Low expression of ethA2 in the absence of EthR2 inhibitors is of clinical importance, offering an explanation as to why EthA2 has not been previously identified as an ETH-bio-activator, and therefore mutations in this gene have not been observed in clinical isolates resistant to ETH so far.

Human-adapted M. tuberculosis complex comprises 7 main phylogenetically distinct lineages (32). We analyzed the genome-sequences of 217 geographically diverse clinical strains representing all seven lineages (33) and confirmed the presence of the rv0076c-rv0078 locus in all strains. In addition, the presence of the rv0076-78 locus was confirmed in a collection of ETH-resistant (171) and ETH-sensitive (253) clinical isolates of M. tuberculosis (34). No mutation in this locus was observed in the ETH sensitive population. Only one ETH resistant isolate shows a point mutation in rv0078, which also contains a Stop mutation in ethA, most probably responsible for the ETH resistance phenotype (Table S3). Then, a panel 
of ETH-sensitive and ETH-resistant MDR M. tuberculosis clinical isolates were tested by respirometry (MGIT 960 ${ }^{\mathrm{TM}}$ (35) ) for their sensitivity to ETH in the presence of SMARt-420. Treatment of ETH-sensitive strains with SMARt-420 $(10 \mu \mathrm{M})$ reduced significantly their MIC for ETH (Table 1, panel C). More importantly, strains highly-resistant to $\mathrm{ETH}$, because of mutations in ethA, were also sensitive to $\mathrm{ETH}$ in the presence of SMARt-420 (Table 1, panel C, Group 4). In conclusion, the combination SMARt-420 plus ETH was active against all ETH-resistant, MDR, and XDR isolates tested. Finally, we showed that overexpression of inhA using a multicopy plasmid modified the boosting effect of SMARt-420, suggesting that the new bioactivation pathway of ETH still target InhA (Fig. S3). We also verified that SMARt-420 used alone does not affect the efficacy of other antibiotics (Table S4).

Pharmacokinetic experiments performed in female Swiss mice showed that a single oral dose of $30 \mathrm{mg} / \mathrm{kg}$ of SMARt-420 provides a circulating concentration of SMARt-420 higher than that required to boost ETH in vitro (Table S5). Restoration of sensitivity to ETH by SMARt-420 was evaluated in C57BL6/J mice infected by aerosol with $10^{5} \mathrm{ETH}$-resistant $M$. tuberculosis bacilli mutated in ethA. Seven days after infection, the mice were treated with ETH alone or with ETH in combination with SMARt-420. Daily administration of up to $50 \mathrm{mg} / \mathrm{kg}$ of ETH for 3 weeks was ineffective in significantly reducing the bacterial load in the lungs (Fig. 4), confirming the resistance to ETH of this strain. In contrast, mice treated with a combination of ETH and SMARt-420 (both at $50 \mathrm{mg} / \mathrm{kg}$ ) showed a striking reduction of the bacterial load (4.6 log) in the lungs (Fig. 4). The absence of an effect observed with SMARt-420 administered alone, and a dose-response to ETH combined with SMARt-420, confirms that the anti-TB activity of the combination is specifically due to the restoration of ETH sensitivity of this strain.

In conclusion, we show that drug-resistance to the widely used antituberculosis drug ETH (36) can be circumvented by spiroisoxazoline SMARt-420 that activates cryptic drug-bioactivation pathways in drugresistant pathogens. Other ETH activation pathways may exist in $M$. tuberculosis, including the recently described VirS-MymA (37), opening supplementary avenues for reversing ETH-resistance and boosting its activity.

Innovative treatment protocols could also be explored, in which non-continuous but periodic administration 
of SMARts to TB-patients would toggle periodically the expression of alternative bio-activation pathways of pro-drugs. This approach could be used to limit the frequency of resistance by systematically destroying subpopulations of resistant bacteria that may emerge during treatment. 


\section{Acknowledgements}

All data and code to understand and assess the conclusions of this research are available in the main text, supplementary materials and via the following repositories: the refined coordinates and the structure factors of Rv0078 were deposited in the Protein Data Bank under the accession numbers 5n1c (iodinated form), 5n1i (unliganded form) and 5icj (liganded form), raw data of RNAseq analysis have been deposited in DRYAD.org under the doi 10.5061/dryad.mb46. We are indebted to the Soleil (BAG proposal 20141408) and the ESRF (BAG proposal MX-1677) synchrotrons for beam-time allocations on this project. We are indebted to Eve Willery for technical support in molecular biology, Florence Leroux for screening management, Laurence Agouridas and Nicolas Probst for chemical synthesis and Catherine Piveteau for analytical pharmacology. This work was supported by l'Agence Nationale de la Recherche, France (Tea4-Two, ANR-14-CE14-0027-01) (ANR-10-EQPX-04-01), by EU grants ERC-STG INTRACELLTB n 260901, the Feder (12001407 (D-AL), Equipex Imaginex BioMed, PRIM (NewBio4Tb), INSERM, Université de Lille, Institut Pasteur de Lille, CNRS, the Région Hauts-de-France (convention n 12000080), SATT-Nord. RW is Research Associate at the National Fund for Scientific Research FNRS-FRS (Belgium). MM was supported by PRIM (NewBio4Tb) and VT by the Marie Curie Initial Training Network (ITN-2013607694-Translocation). The authors also thank the UMR 8199 LIGAN-PM Genomics platform (Lille, France) which belongs to the 'Federation de Recherche' 3508 Labex EGID (European Genomics Institute for Diabetes; ANR-10-LABX-46) and was supported by the ANR Equipex 2010 session (ANR-10-EQPX07-01; 'LIGAN-PM'). The LIGAN-PM Genomics platform is supported by the FEDER and the Region NordPas-de-Calais-Picardie. We thank Agnès Wagner and Roquette Frères (Lestrem, France) for their gift of Cyclodextrine Kleptose HP. We greatly appreciate the fruitful discussions with all the members of the GlaxoSmithKline team "Diseases of the Developing World (DDW)" and their invaluable support in the continuation of this project. CK declare to be employed at Bioversys and to own stock options of the company, SB is a consultant for the Foundation of Innovative Diagnostics (FIND) in Geneva, Switzerland. MG is CEO of Bioversys and a Board Member of the BEAM Alliance, a group of Biopharmaceutical companies from Europe innovating in Anti-Microbial resistance research. The BEAM Alliance is a not for 
profit association and there is no financial remuneration of any kind for its Board members. All companies included are working within the field of antimicrobial drug discovery. NW, BD, ARB, PB, MD, are inventors on patent PCT/EP2013/077706 that covers BDM41420; WO/2014/096369. BD, NW, CL, ARB are inventors on patent PCT/FR2007/001138; WO/2008/003861 that covers SMARt-420 (BDM41420). The facilities conformed to Directive 86/609/EEC on the Protection of Animals Used for Experimental and Other Scientific Purposes and norms published in the European Council ETS123 Appendix A. Facilities and procedures complied with the Belgian Law of 14th of August 1986 on animal protection and welfare. Training of experimental leaders, bio-technicians and animal care-takers was in accordance with Royal decree of 13/9/2004 which specifies the training of persons working with laboratory animals. All animal experimentation and procedures performed at the National Reference Center for Tuberculosis and Mycobacteria, Bacterial Diseases Service, Scientific Institute of Public Health (WIV-ISP), Brussels, Belgium, were validated and approved by the Ethical Committee of the IPH-VAR (Belgium) under the file number 120323-01. The animal facilities and procedures were under the supervision of an expert on animal welfare in accordance with the Belgian Ministry of Health. pET-15b-ethR2, $p E T-15 b$-ethR are available from ARB under a material transfer agreement with Institut Pasteur de Lille. pCK289, pCK287, are available from MG under a material transfer agreement with Bioversys. 


\section{References}

1. B. Spellberg, D. N. Gilbert, The future of antibiotics and resistance: a tribute to a career of leadership by John Bartlett. Clin. Infect. Dis. 59 Suppl 2, S71-75 (2014).

2. WHO, Antimicrobial resistance: Global report on surveillance. Bull. W.H.O., (2014).

3. S. Hoffner, Unexpected high levels of multidrug-resistant tuberculosis present new challenges for tuberculosis control. Lancet 380, 1367-1369 (2012).

4. WHO, Global tuberculosis report. World Health Organization, Geneva, Switzerland, (2015).

5. C. Barry, Infectious disease. More than just bugs in spit. Science 348, 633-634 (2015).

6. C. Walsh, Antibiotics: actions, origins, resistance. (ASM Press, Washington, D.C, 2003).

7. C. Reading, M. Cole, Clavulanic acid: a beta-lactamase-inhiting beta-lactam from Streptomyces clavuligerus. Antimicrob. Agents Chemother. 11, 852-857 (1977).

8. Y. Zhang, B. Heym, B. Allen, D. Young, S. Cole, The catalase peroxidase gene and isoniazid resistance of Mycobacterium tuberculosis. Nature 358, 591-593 (1992).

9. A. Scorpio, Y. Zhang, Mutations in pncA, a gene encoding pyrazinamidase/nicotinamidase, cause resistance to the antituberculous drug pyrazinamide in tubercle bacillus. Nat. Med. 2, 662-667 (1996).

10. A. E. DeBarber, K. Mdluli, M. Bosman, L. G. Bekker, C. E. Barry, 3rd, Ethionamide activation and sensitivity in multidrug-resistant Mycobacterium tuberculosis. Proc. Natl. Acad. Sci. U. S. A. 97, 9677-9682 (2000).

11. A. R. Baulard et al., Activation of the pro-drug ethionamide is regulated in mycobacteria. J. Biol. Chem. 275, 28326-28331 (2000).

12. U. H. Manjunatha et al., Identification of a nitroimidazo-oxazine-specific protein involved in PA-824 resistance in Mycobacterium tuberculosis. Proc. Natl. Acad. Sci. U. S. A. 103, 431-436 (2006).

13. M. Matsumoto et al., OPC-67683, a nitro-dihydro-imidazooxazole derivative with promising action against tuberculosis in vitro and in mice. PLoS medicine 3, e466 (2006).

14. G. V. Bloemberg et al., Acquired Resistance to Bedaquiline and Delamanid in Therapy for Tuberculosis. N. Engl. J. Med. 373, 1986-1988 (2015).

15. J. Zheng et al., para-Aminosalicylic acid is a prodrug targeting dihydrofolate reductase in Mycobacterium tuberculosis. J. Biol. Chem. 288, 23447-23456 (2013).

16. T. A. Vannelli, A. Dykman, P. R. Ortiz De Montellano, The antituberculosis drug ethionamide is activated by a flavoprotein monooxygenase. J. Biol. Chem. 277, 12824-12829. (2002).

17. X. Hanoulle et al., Selective intracellular accumulation of the major metabolite issued from the activation of the prodrug ethionamide in mycobacteria. J. Antimicrob. Chemother. 58, 768-772 (2006).

18. F. Wang et al., Mechanism of thioamide drug action against tuberculosis and leprosy. J. Exp. Med. 204, 73-78 (2007).

19. A. Banerjee et al., inhA, a gene encoding a target for isoniazid and ethionamide in Mycobacterium tuberculosis. Science 263, 227-230 (1994).

20. J. Engohang-Ndong et al., EthR, a repressor of the TetR/CamR family implicated in ethionamide resistance in mycobacteria, octamerizes cooperatively on its operator. Mol. Microbiol. 51, 175-188 (2004).

21. N. Willand et al., Exploring drug target flexibility using in situ click chemistry: application to a mycobacterial transcriptional regulator. ACS Chem. Biol. 5, 1007-1013 (2010).

22. M. Flipo et al., Ethionamide boosters: synthesis, biological activity, and structure-activity relationships of a series of 1,2,4-oxadiazole EthR inhibitors. J. Med. Chem. 54, 2994-3010 (2011).

23. M. Flipo et al., Ethionamide boosters. 2. Combining bioisosteric replacement and structure-based drug design to solve pharmacokinetic issues in a series of potent 1,2,4-oxadiazole EthR inhibitors. J. Med. Chem. 55, 68-83 (2012).

24. M. Flipo et al., Discovery of novel N-phenylphenoxyacetamide derivatives as EthR inhibitors and ethionamide boosters by combining high-throughput screening and synthesis. J. Med. Chem. 55, 6391-6402 (2012).

25. N. Willand et al., Synthetic EthR inhibitors boost antituberculous activity of ethionamide. Nat. Med. 15, 537-544 (2009). 
26. L. Cuthbertson, J. R. Nodwell, The TetR family of regulators. Microbiol. Mol. Biol. Rev. 77, 440475 (2013).

27. M. A. Schumacher et al., Structural mechanisms of QacR induction and multidrug recognition. Science 294, 2158-2163. (2001).

28. F. Frenois, J. Engohang-Ndong, C. Locht, A. R. Baulard, V. Villeret, Structure of EthR in a ligand bound conformation reveals therapeutic perspectives against tuberculosis. Mol. Cell 16, 301-307 (2004).

29. F. Frenois, A. R. Baulard, V. Villeret, Insights into mechanisms of induction and ligands recognition in the transcriptional repressor EthR from Mycobacterium tuberculosis. Tuberculosis (Edinb) 86, 110-114 (2006).

30. W. Weber et al., A synthetic mammalian gene circuit reveals antituberculosis compounds. Proc. Natl. Acad. Sci. U. S. A. 105, 9994-9998 (2008).

31. C. K. Stover et al., New use of BCG for recombinant vaccines. Nature 351, 456-460 (1991).

32. M. Coscolla, S. Gagneux, Consequences of genomic diversity in Mycobacterium tuberculosis. Semin. Immunol. 26, 431-444 (2014).

33. I. Comas et al., Out-of-Africa migration and Neolithic coexpansion of Mycobacterium tuberculosis with modern humans. Nat. Genet. 45, 1176-1182 (2013).

34. N. Casali et al., Evolution and transmission of drug-resistant tuberculosis in a Russian population. Nat. Genet. 46, 279-286 (2014).

35. F. Ardito, B. Posteraro, M. Sanguinetti, S. Zanetti, G. Fadda, Evaluation of BACTEC Mycobacteria Growth Indicator Tube (MGIT 960) automated system for drug susceptibility testing of Mycobacterium tuberculosis. J. Clin. Microbiol. 39, 4440-4444 (2001).

36. S. D. Ahuja et al., Multidrug resistant pulmonary tuberculosis treatment regimens and patient outcomes: an individual patient data meta-analysis of 9,153 patients. PLoS medicine 9, e1001300 (2012).

37. S. S. Grant et al., Baeyer-Villiger Monooxygenases EthA and MymA Are Required for Activation of Replicating and Non-replicating Mycobacterium tuberculosis Inhibitors. Cell Chem Biol 23, 666677 (2016).

38. M. B. Sauton, Sur la nutrition minérale du bacille tuberculeux. C R Acad Sci Paris 155, 860-861 (1912).

39. W. Kabsch, Xds. Acta Crystallogr. D. Biol. Crystallogr. 66, 125-132 (2010).

40. A. Vagin, A. Teplyakov, Molecular replacement with MOLREP. Acta Crystallogr. D. Biol. Crystallogr. 66, 22-25 (2010).

41. A. A. Vagin et al., REFMAC5 dictionary: organization of prior chemical knowledge and guidelines for its use. Acta Crystallogr. D. Biol. Crystallogr. 60, 2184-2195 (2004).

42. M. D. Winn et al., Overview of the CCP4 suite and current developments. Acta Crystallogr. D. Biol. Crystallogr. 67, 235-242 (2011).

43. P. Emsley, B. Lohkamp, W. G. Scott, K. Cowtan, Features and development of Coot. Acta Crystallogr. D. Biol. Crystallogr. 66, 486-501 (2010).

44. S. Schlatter, M. Rimann, J. Kelm, M. Fussenegger, SAMY, a novel mammalian reporter gene derived from Bacillus stearothermophilus alpha-amylase. Gene 282, 19-31 (2002).

45. V. Delorme, O. R. Song, A. Baulard, P. Brodin, Testing chemical and genetic Modulators in Mycobacterium tuberculosis infected cells using phenotypic assays. Methods Mol. Biol. 1285, 387-411 (2015). 


\begin{tabular}{|c|c|c|c|c|c|c|c|c|c|}
\hline \multicolumn{8}{|c|}{ Panel A } & \multirow{2}{*}{$\begin{array}{c}\text { Panel B } \\
\begin{array}{c}\text { ETH } \\
+\end{array} \\
\text { BDM } \\
41906\end{array}$} & \multirow{2}{*}{$\begin{array}{c}\text { Panel C } \\
\begin{array}{c}\text { ETH } \\
+\end{array} \\
\begin{array}{c}\text { SMARt } \\
420\end{array}\end{array}$} \\
\hline $\begin{array}{c}\text { TB } \\
\text { strains }\end{array}$ & $\begin{array}{c}\mathrm{INH} \\
\\
0.1 \\
\mu \mathrm{g} / \mathrm{mI}\end{array}$ & $\begin{array}{c}\text { RIF } \\
\begin{array}{c}1 \\
\mu g / m I\end{array}\end{array}$ & $\begin{array}{c}\text { EMB } \\
\\
5 \\
\mu \mathrm{g} / \mathrm{ml}\end{array}$ & $\begin{array}{c}\text { OFL } \\
1 \\
1 \\
\mu \mathrm{g} / \mathrm{ml}\end{array}$ & $\begin{array}{c}\text { AMI } \\
1 \\
\mu \mathrm{g} / \mathrm{ml}\end{array}$ & $\mid \begin{array}{c}\text { ETH } \\
5 \\
\mu \mathrm{g} / \mathrm{ml}\end{array}$ & $\begin{array}{c}\text { EthA } \\
\text { status }\end{array}$ & & \\
\hline \multicolumn{10}{|c|}{ Group 1. Pan-susceptible laboratory strain } \\
\hline H37Rv & & & & & & 2 & wt & 0,01 & 0,05 \\
\hline \multicolumn{10}{|c|}{ Group 2. Ethionamide sensitive - MDR strains } \\
\hline L4376 & & & & & & 4 & wt & 0,1 & 0,05 \\
\hline LPN30 & & & & & & 2 & nd & 0,1 & 0,05 \\
\hline L1094 & & & & & & 2 & wt & 0,1 & 0,05 \\
\hline P591 & & & & & & 2 & nd & 0,1 & 0,05 \\
\hline L0578 & & & & & & 1 & wt & 0,05 & 0,25 \\
\hline \multicolumn{10}{|c|}{ Group 3. Ethionamide resistant - EthA wt - MDR strains } \\
\hline B1166 & & & & & & 64 & wt & 4 & 0,05 \\
\hline B1196 & & & & & & 32 & wt & 0,25 & 0,25 \\
\hline B1004 & & & & & & 32 & wt & 0,25 & 0,5 \\
\hline \begin{tabular}{|l} 
B1304 \\
\end{tabular} & & & & & & 32 & wt & 1 & 0,025 \\
\hline P379 & & & & & & 32 & wt & 4 & 0,05 \\
\hline \begin{tabular}{|l} 
B1001 \\
\end{tabular} & & & & & & 32 & wt & 4 & 0,05 \\
\hline B0383 & & & & & & 16 & wt & 1 & 0,5 \\
\hline B0391 & & & & & & 8 & wt & 0,1 & 0,25 \\
\hline B0089 & & & & & & 8 & wt & 0,25 & 0,5 \\
\hline \begin{tabular}{|l|} 
LPN4 \\
\end{tabular} & & & & & & 8 & wt & 0,5 & 0,025 \\
\hline P395 & & & & & & 8 & wt & 1 & 0,025 \\
\hline P359 & & & & & & 8 & wt & 2 & 0,05 \\
\hline P351 & & & & & & 8 & wt & 2 & 0,05 \\
\hline \multicolumn{10}{|c|}{ Group 4. Ethionamide resistant - EthA mutated - MDR strains } \\
\hline B1150 & & & & & & 256 & $\mathrm{P} 230 \mathrm{Q}$ & $>64$ & 0,5 \\
\hline B1602 & & & & & & 256 & R239G & $>64$ & 0,5 \\
\hline B0775 & & & & & & 256 & $\Delta \mathrm{a} 110$ & 32 & 0,25 \\
\hline B0057 & & & & & & 64 & $\Delta a 110$ & 64 & 0,25 \\
\hline L3556 & & & & & & 64 & Q165P & 64 & 0,5 \\
\hline L0728 & & & & & & 64 & ins.c357 & 8 & 0,25 \\
\hline
\end{tabular}

\section{Table 1.}

Impact of BDM41906 and SMARt-420 on the ethionamide susceptibility of a selection of clinical strains.

Panel A. Antibiotic profile. Threshold concentrations above which bacteria are considered clinically resistant are indicated. The drug sensitivity status of each strain is reported, green color meaning "under the threshold concentration", and red color meaning "above the threshold concentration". Specifically, for ETH, Minimal Inhibitory Concentrations have been defined by MGIT960 and are reported (values in $\mu \mathrm{g} / \mathrm{ml}$ ). All selected strains except the reference pan-susceptible laboratory strain H37Rv (Group 1) are multidrugresistant (INH and RIF resistant). Group 2 includes ETH sensitive strains. Group 3 contains ETH resistant strains without mutation in ethA. Group 4 contains ETH resistant strains mutated in ethA. Panel B. MIC of $\mathrm{ETH}$ in the presence of $10 \mu \mathrm{M}$ of first generation booster BDM41906. Panel C. MIC of ETH in the presence of $10 \mu \mathrm{M}$ SMARt-420. 


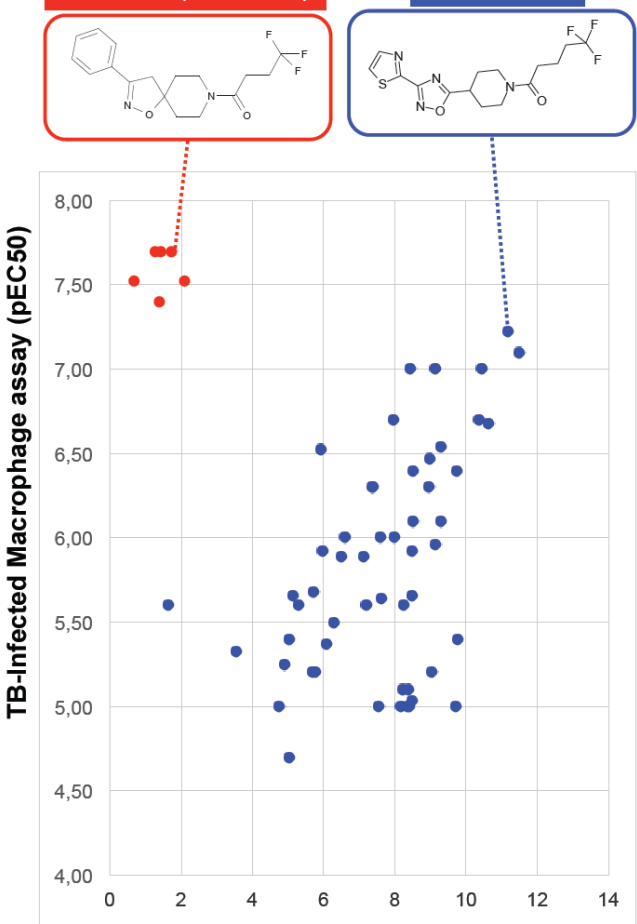

Thermal Shift Assay on EthR $\left(\Delta \mathrm{Tm}\right.$ in $\left.{ }^{\circ} \mathrm{C}\right)$

\begin{tabular}{|c|c|c|c|c|c|c|}
\hline \multicolumn{2}{|c|}{ Gene Name } & $\begin{array}{c}(1) \\
\text { RPKM } \\
\text { control } \\
\end{array}$ & $\begin{array}{c}(2) \\
\text { RPKM } \\
41906 \\
\end{array}$ & $\begin{array}{c}(2) /(1) \\
\text { FC }\end{array}$ & $\begin{array}{c}(3) \\
\text { RPKM } \\
\text { Smart420 }\end{array}$ & $\begin{array}{c}\text { (3)/(1) } \\
\text { FC }\end{array}$ \\
\hline $\begin{array}{l}\text { ethA } \\
\text { ethR }\end{array}$ & BCG_3917c & 74 & 2250 & 30,41 & $\begin{array}{c}184 \\
89\end{array}$ & 2,49 \\
\hline mmpS5 & BCG $0726 \mathrm{C}$ & 111 & $\begin{array}{l}653 \\
263\end{array}$ & 2,37 & 107 & $\begin{array}{c}2,54 \\
\text { ns }\end{array}$ \\
\hline 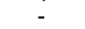 & BCG_0108c & 23 & 57 & 2,48 & 6186 & 268,96 \\
\hline - & BCG_0107c & 47 & 50 & ns & 4016 & 85,45 \\
\hline - & BCG_0269 & 89 & 157 & 1,76 & 242 & 2,72 \\
\hline - & BCG_1777 & 50 & 10 & ns & 126 & 2,52 \\
\hline $\operatorname{nrdB}$ & BCG_0270 & 78 & 125 & 1,60 & 188 & 2,41 \\
\hline $\mathrm{mmpS} 4$ & BCG_0490c & 127 & 110 & ns & 265 & 2,09 \\
\hline
\end{tabular}

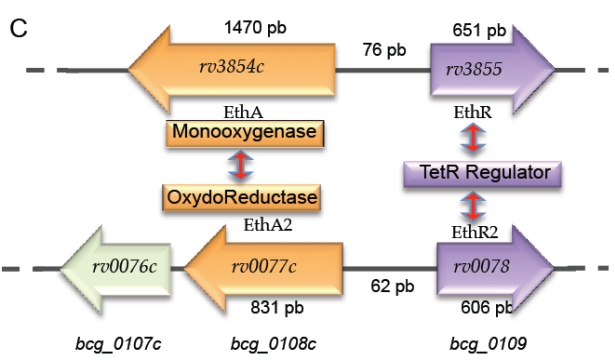

Figure 1. SMARt family of molecules reveals alternative ETH bioactivation pathway

(A). Bidimentionnal representation of the properties of ETH-boosting compounds (BDM). The x-axis indicates the shift in the melting temperature $(\Delta \mathrm{Tm})$ of EthR in the presence of BDM compounds, which translates the capacity of the compounds to bind and thermostabilize EthR in vitro (see Table S1 for values). The $y$-axis indicates the potency (expressed as the negative logarithm of the EC50) of a panel of compounds to increase ethionamide antibacterial activity on M. tuberculosis infected macrophages. EC50 is the concentration of compound that allows ethionamide at $0.1 \mu \mathrm{g} / \mathrm{mL}$ (10 times less than the normal MIC) to inhibit $50 \%$ of $M$. tuberculosis growth in macrophages. Blue dots and red dots represent compounds of the oxadiazole-piperidine family (first generation boosters) and of the spiroisoxazoline family (SMARt), respectively. (B). RNA-seq analysis of genes that are differentially expressed in $M$. bovis BCG exposed for 24 hours to $25 \mu \mathrm{M}$ BDM41906 (2) or SMARt-420 (3) in comparison to DMSO treated bacteria (1). Only genes showing a minimum 2-fold change $(F C)$ in transcript abundance in at least one condition are shown. RPKM: Reads Per Kilobase per Million mapped reads. Whereas BDM41906 specifically induce the expression of ethA and ethR, SMARt-420 massively induces the expression of bcg_0108c and bcg_0107c. A weak, but statistically significant, induction of ethA and ethR is also observed. (C). Comparison of the genetic organization and predicted function of the corresponding M. tuberculosis loci and proteins. Genes bcg_0107c and bcg_108c correspond to rv0076c and rv0077c, respectively. Rv0078 is predicted as a transcriptional repressor of the TetR family. 
A

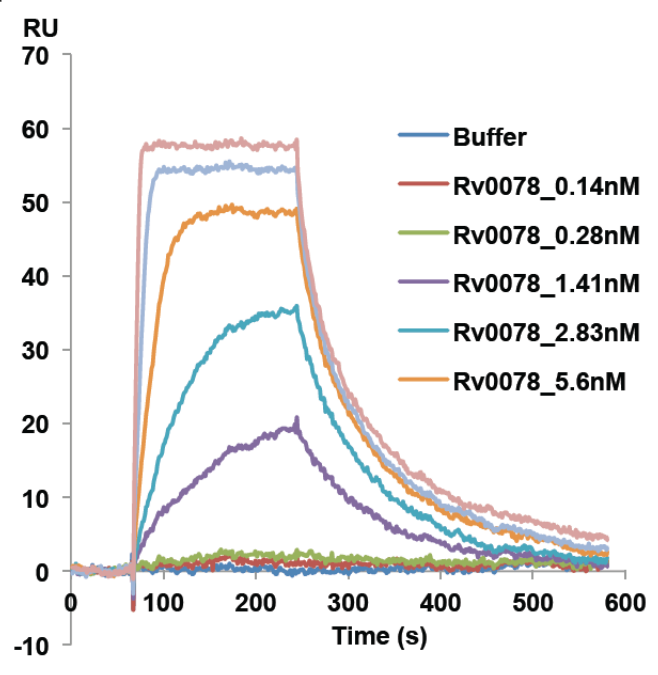

B

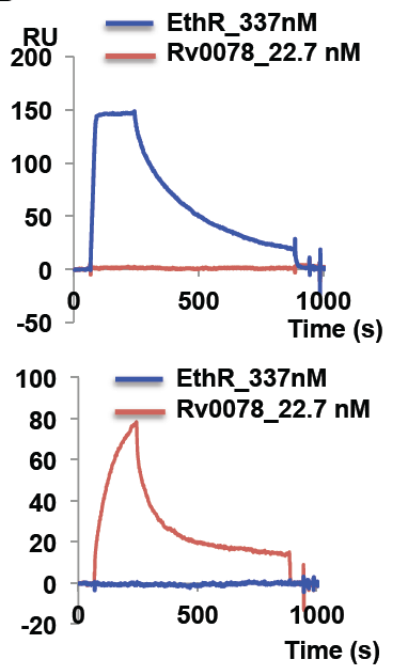

C

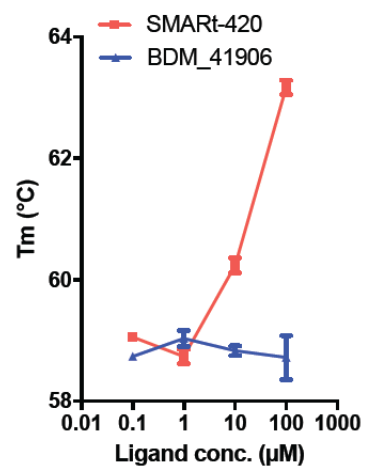

$\mathrm{D}$

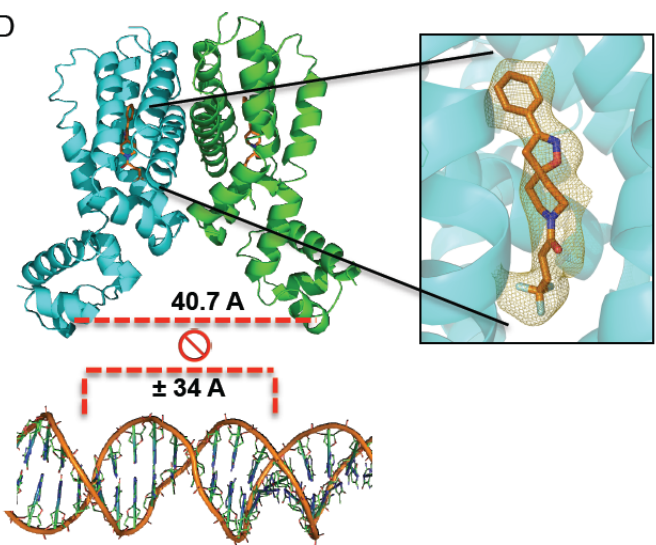

Figure 2. SMARt-420 interacts with the transcriptional regulator EthR2.

(A). Global affinity of the interaction between EthR2 and $P_{\text {ethR2 }}$ measured by SPR. Sensorgrams of 22.7, 11.35, 5.60, 2.83, 1.41 and $0.28 \mathrm{nM}$ EthR2 (Rv0078) injections over a Sensor Chip functionalized with $40 \mathrm{RU}$ (resonance unit) of the biotinylated ethA2-ethR2 intergenic DNA.

(B). Comparison of the injection of EthR and EthR2 (Rv0078) over 2 Sensor Chips functionalized with ethA-ethR intergenic DNA (top) and with ethA2-R2 intergenic DNA (bottom), respectively.

(C). Binding of SMARt-420 and BDM41906 on EthR2 (Rv0078) measured by determination of the melting temperature $(\mathrm{Tm})$ of the complex (Thermal-shift assay, see Material and Methods).

(D). Crystal structure of the EthR2/SMARt-420 complex and illustration of the steric inability of binding of the repressor to DNA due to the 40.7A spacing of the HTH motifs. The zoom onto the ligand binding pocket of the protein shows the electron density (Fo-Fc) omit map contoured at 1.2 sigma around one of the two SMARt-420 molecules embedded in each monomer of the EthR2 homodimer (see Supplementary Material \& Methods and Table S2 for statistics). 
A
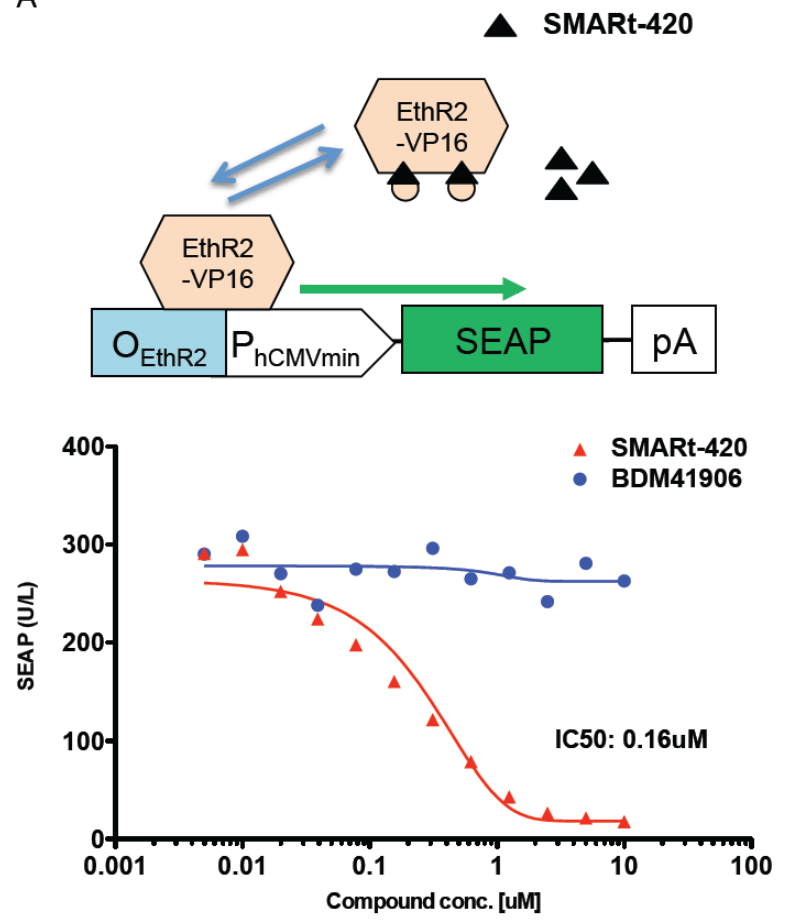

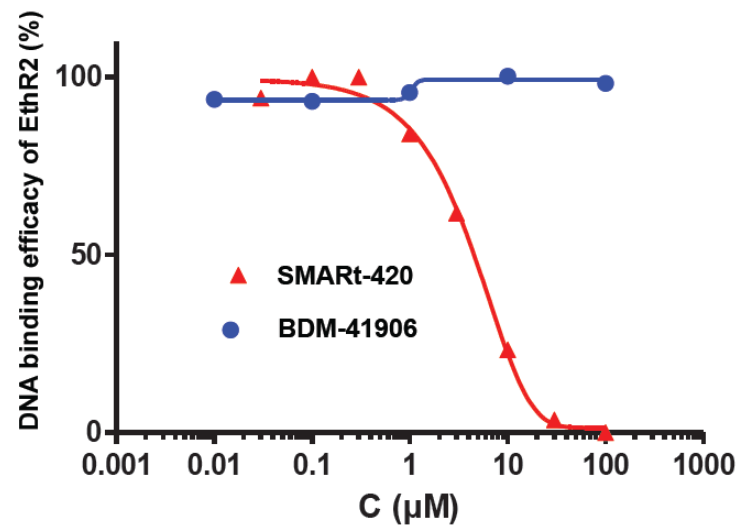

C

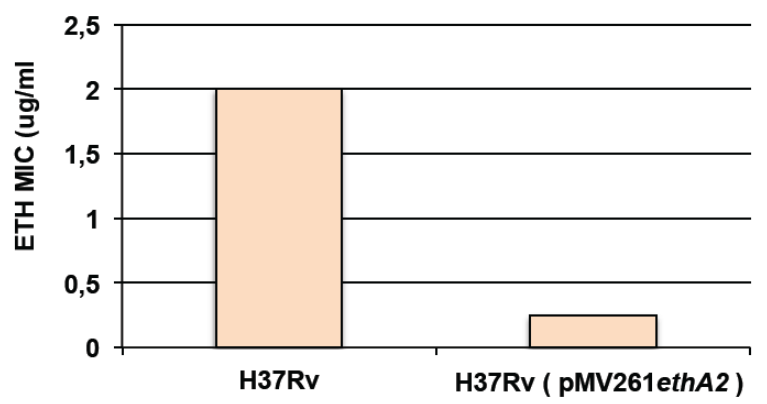

Figure 3. SMARt-420 inhibits the DNA binding activity of EthR2.

(A). Synthetic mammalian gene circuit designed to sense EthR2-DNA interactions (ethA2-ethR2 intergenic region) that are required to induce expression of the reporter gene SEAP. The alkaline phosphatase induced by the EthR2-VP16 complex was inhibited in a dose-dependent manner by SMARt420, whereas no effect was observed in the presence BDM41906 (see Supplementary Material \& Methods and Fig. S1).

(B). Inhibition of the binding of EthR2 to the ethA2-ethR2 intergenic region by SMARt-420 but not by BDM41906 measured by surface plasmon resonance.

(C). Effect of the overexpression of ethA2 (pMV261-ethA2) on the minimal inhibitory concentration of ethionamide in M. tuberculosis. 


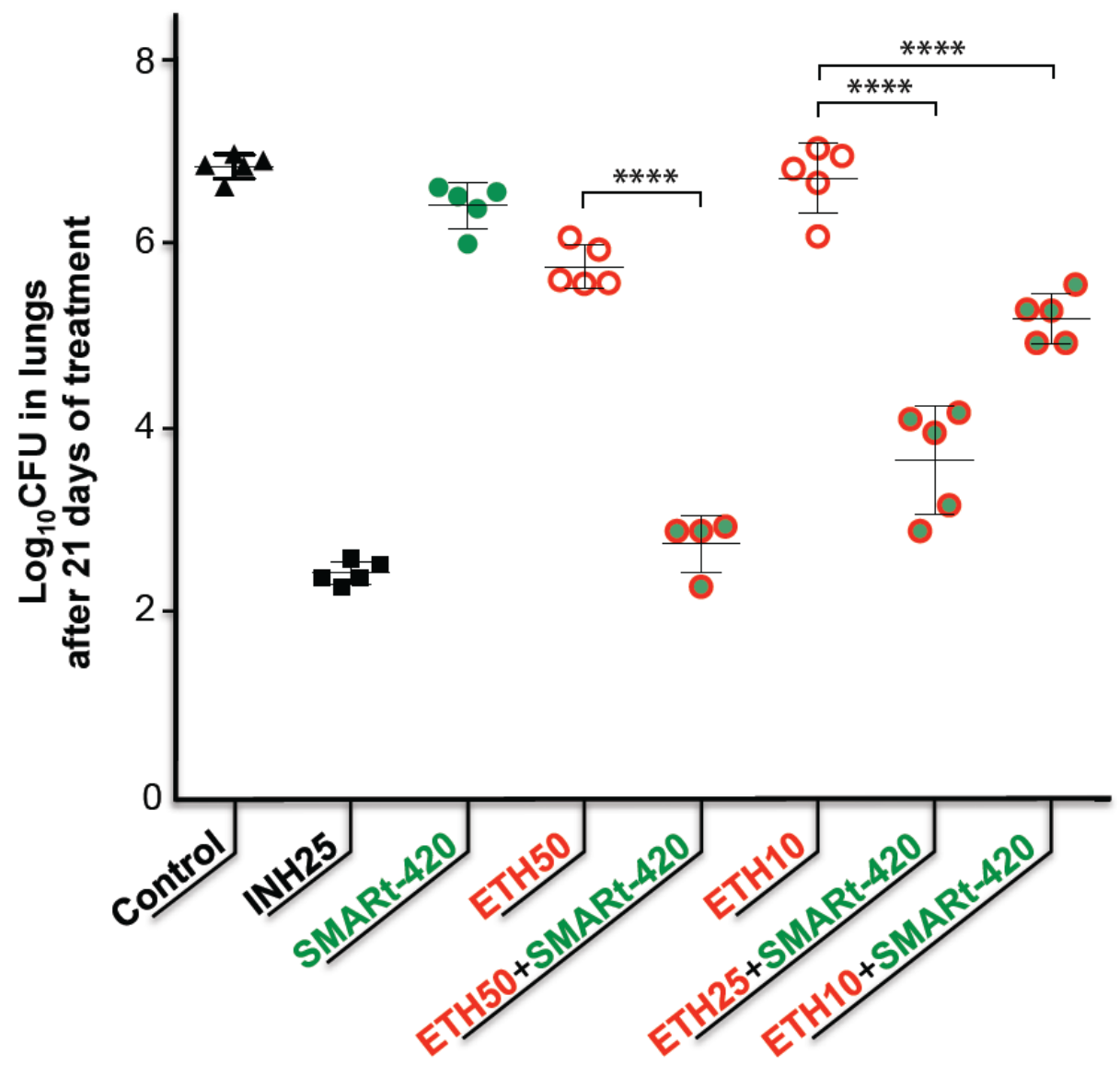

Figure 4. Reversion of ETH-resistance in tuberculosis infected mice.

Mice (5 mice per group) infected with ETH-resistant bacteria were treated by the control antibiotic INH (25mg/kg), ETH alone (50mg/kg), SMARt-420 alone $(50 \mathrm{mg} / \mathrm{kg})$, or a combination of ETH and SMARt-420. Pulmonary bacillary loads were enumerated by CFUs after 3 weeks of treatment. Administration of up to $50 \mathrm{mg} / \mathrm{kg}$ of ETH did not reduce the pulmonary load of ETH-resistant mycobacteria, whereas coadministration of ETH and SMARt-420 showed a dose dependent reduction with a maximum of 4.6 log (Control versus ETH50+SMARt-420). See "Bonferroni's multiple comparison test" in Table S6 for details and statistics. 


\section{Supplementary Material}

The Supplementary Material PDF file includes:

Materials and Methods

Figs. S1 to S5

Tables S1 to S6

Captions for databases S1

Other Supplementary Materials for this manuscript includes:

DRYAD.org link to the zipped archive "Databases S1.tar.gz" containing RnaSeq results for :

$$
\begin{aligned}
& \text { BCG_DMSO.fastq } \\
& \text { BCG_BDM41906.fastq } \\
& \text { BCG_SMARt-420.fastq }
\end{aligned}
$$

Table S3.xlsx

References (38-45) 